\title{
Neuroticismo em Pacientes com Transtorno da Personalidade Borderline Hospitalizados
}

\author{
Letícia Garibaldi Gasparetto ${ }^{1}$, Claudio Simon Hutz \\ Universidade Federal do Rio Grande do Sul, Porto Alegre-RS, Brasil
}

\section{RESUMO}

O objetivo deste trabalho foi investigar as diferenças entre os sexos na Escala Fatorial de Neuroticismo (EFN) e em suas facetas em 40 pacientes com Transtorno da Personalidade Borderline internados em uma clínica psiquiátrica bem como a ocorrência de TEPT nesses pacientes. Também foi aplicada a Borderline Symptoms List (BSL-23) para avaliar a sintomatologia do Transtorno Borderline. Os resultados mostraram uma pontuação elevada dessa amostra na EFN com relação a amostra de normatização da escala $(122,39 \pm 12,00)$. Não houve diferença significativa entre os sexos. Muitos pacientes apresentaram como comorbidade o Transtorno Depressivo Maior (40\%) e tanto homens quanto mulheres obtiveram as pontuações mais elevadas na faceta depressão. Os resultados indicaram a ocorrência de experiências traumáticas na infância e a probabilidade cinco vezes maior de pessoas com TPB com histórico de abuso de desenvolverem TEPT. Sugere-se um estudo com populações clínicas maiores, na tentativa de encontrar um ponto de corte ou um padrão de pontuação nos instrumentos de Neuroticismo que possa indicar TPB e outros estudos que aprofundem as relações entre TEPT, abuso e a ocorrência de depressão nesses pacientes.

Palavras-chave: transtorno de personalidade; borderline; neuroticismo.

\section{ABSTRACT - Neuroticism in Hospitalized Patients with Boderline Personality Disorder}

The objective of this study was to investigate the differences between the sexes in the Factorial Neuroticism Scale (FNS) and its facets in 40 patients with borderline personality disorder hospitalized in a psychiatric clinic, as well as the occurrence of PTSD in these patients. The Borderline Symptoms List (BSL-23) was also applied to evaluate the symptomatology of borderline personality disorder. The results showed a high FNS score in this sample in relation to the scale's normalization sample $(122.39 \pm 12.00)$. There was no significant difference between the sexes. Many patients presented Major Depressive Disorder (40\%) as comorbidity, and both men and women had the higher scores in the depression facet. The results indicated the occurrence of traumatic experiences in childhood and a five-fold higher probability of people with BPD and a history of abuse of developing PTSD. We suggest a study with larger clinical populations in an attempt to find a cutoff point or a scoring standard in the Neuroticism instrument that could indicate BPD, and other studies that deepen the relations between PTSD, abuse and the occurrence of Depression in these patients.

Keywords: Personality Disorder; Borderline; Neuroticism.

\section{RESUMEN - Inestabilidad Emocional en Pacientes Hospitalizados con Trastorno de Personalidad Bordeline}

El objetivo de este trabajo fue investigar las diferencias entre los sexos en la Escala Factorial de Inestabilidad Emocional EFN y en sus facetas, en 40 pacientes con trastorno de personalidad bordeline internados en una clínica psiquiátrica así como la ocurrencia de TEPT en los mismos. También se aplicó la Borderline Symptoms List (BSL-23) para evaluar la sintomatología del trastorno bordeline. Los resultados mostraron alta puntuación en esta muestra en la EFN con relación a la muestra de normatización de la escala $(122,39 \pm 12,00)$. No se dieron diferencias significativas entre los sexos. Muchos pacientes presentaron como comorbidad el Trastorno Depresivo Mayor (40\%) y tanto hombres como mujeres obtuvieron las puntuaciones más elevadas en la faceta depresión. Los resultados indicaron la ocurrencia de experiencias traumáticas en la infancia y la probabilidad cinco veces mayor de personas con TPB con histórico de abuso, de desarrollar TEPT. Se sugiere un estudio con poblaciones clínicas mayores, tratando de encontrar un punto de corte o un patrón de puntuación en los instrumentos de Inestabilidad Emocional que puedan indicar TPB y otros estudios para investigar más a fondo la relación entre el trastorno de estrés postraumático TEPT, abuso y la ocurrencia de depresión en esos pacientes.

Palabras clave: Trastorno de Personalidad; Borderline; Inestabilidad Emocional.

O Transtorno da Personalidade Borderline (TPB) é o mais prevalente dentre os transtornos da personalidade na população geral como primeiro diagnóstico (Torgersen, 2009) e na população clínica já diagnosticada com algum outro transtorno de personalidade (Zimmerman, Rothschild, \& Chelminski, 2005) e atinge cerca de $6 \%$ dos pacientes em cuidados primários, $10 \%$ dos pacientes em ambulatórios psiquiátricos e $20 \%$ entre os que se 
hospitalizararam em unidades psiquiátricas (American Psychiatric Association, 2013). O TPB é associado a significativo prejuízo físico e mental e, em alguns casos, necessidade de tratamento medicamentoso, atendimento ambulatorial, internação e um alto risco de mortalidade por suicídio (Grant et al., 2008), já que, dentre os pacientes, $10 \%$ cometem suicídio e $75 \%$ já tiveram pelo menos uma tentativa (Soloff et al., 2005).

A etiologia do transtorno tem sido investigada e debatida por décadas (Gunderson, 2009). Os atuais modelos etiológicos multifatoriais destacam as interações de fatores psicossociais, genéticos e neurobiológicos na gênese do TPB (Leichsenring et al.,2011). Entre os fatores psicossociais, experiências traumáticas na infância e apego inseguro têm recebido grande suporte empírico (Mosquera et al., 2014). Pacientes com TPB tendem a reportar mais situações traumáticas do que outros pacientes psiquiátricos e a maioria dessas situações são de abuso sexual (Yen et al., 2002; Linehan, 2009).

Para diagnosticar transtornos de personalidade, existem modelos prototípicos, híbridos, categóricos, dimensionais, entre outros. No modelo categórico, sintomatologia e diagnóstico são apontados como entidades discretas, ou seja, elas têm caráter de presença/ausência. Já o modelo dimensional aceita uma variedade de dificuldades e decisões ambíguas para cada paciente (Widiger, 1993), permitindo distinções categóricas quando necessário e esclarecendo ao terapeuta o grau no qual um estilo de personalidade não é adaptativo dentro de um continuum ou a extensão no qual cada desordem de personalidade está presente.

Estudos demonstram que clínicos consistentemente indicam modelos dimensionais, como o modelo dos Cinco Grandes Fatores, como mais úteis do que as categorias diagnósticas categóricas dos manuais diagnósticos e estatísticos de transtornos mentais (Livesley, 2007; Brow \& Barlow, 2009, Krueger et al., 2011). Os Cinco Grandes Fatores (CGF) têm recebido um amplo suporte nas últimas décadas, em razão de pesquisas sobre a universalidade dessas dimensões e da validação com inventários de traços e outros questionários e instrumentos de avaliação reconhecidos no meio científico (Pervin \& John, 2004). Costa e McCrae (2007) afirmam que os cinco fatores representam as dimensões mais básicas identificadas tanto na linguagem natural quanto em questionários psicológicos.

Em um estudo realizado por Costa e McCrae (2007), utilizando o NEO-PI-R (escala utilizada para avaliação da personalidade a partir do modelo do CGF), o instrumento indicou replicabilidade em seis línguas diferentes. Pesquisadores têm documentado que traços de personalidade oriundos dos CGF e aqueles subjacentes aos transtornos de personalidade do DSM-IV (O'Connor, 2002; Markon et al., 2005) e do DSM-5 (Chiu, 2013; Gore, 2013) coincidem na sua estrutura. Os
Cinco Grandes Fatores são: Neuroticismo, Extroversão, Amabilidade, Conscienciosidade e Abertura à novas experiências. O Neuroticismo é o fator associado às características emocionais das pessoas, razão pela qual será enfocado no presente estudo. Um alto nível de Neutoticismo identifica indivíduos que são propensos a vivenciar mais intensamente o sofrimento emocional. Tais pessoas tendem a ser facilmente frustráveis, incapazes de resistir aos impulsos e de lidar com o estresse, mais tensos, preocupados, apreensivos, melancólicos, tristes, com tendências a se culpabilizar e a possuir sentimentos de vergonha, inferioridade e constrangimento. Níveis extremos de Neuroticismo, muito elevados ou muito baixos, podem ser indicadores de padrões pouco adaptativos (McCrae \& John, 1992).

Estudos buscaram verificar a relação entre transtornos da personalidade identificados a partir dos critérios propostos pelo DSM-IV e avaliações realizadas no modelo dos Cinco Grandes Fatores (Samuel \& Widiger, 2008; Chmielewski et al., 2011). Nestes e em outros estudos (Wischniewski \& Brüne, 2013; Saulsman \& Page, 2004), os pacientes com TPB apresentaram em comum escores bastante elevados no fator Neuroticismo e escores baixos em Amabilidade e Conscienciosidade.

A pontuação elevada no fator Neuroticismo é expressiva e recorrente em diversos transtornos psíquicos (Wischniewski \& Brüne, 2013; Widiger \& Samuel, 2008; Saulsman \& Page, 2004). Contudo, esse fator é muito característico de pacientes com TPB, permitindo, inclusive, distinguir aqueles que possuem o transtorno daqueles que não o possuem, conforme indicam Morey e Zanarini (2000). Estima-se que a herdabilidade para o fator Neuroticismo em pacientes Borderline seja de $43 \%$ e que há correlações fenotípicas de 0,68 (Distel et al., 2009).

Estudo dos transtornos da personalidade com os CGF têm concluído que análises focadas nas facetas dos fatores do que propriamente nestes são mais eficazes em capturar os construtos subjacentes aos transtornos da personalidade (Samuel \& Widiger, 2008; Clark, 2007; Bagby et al., 2005). Em razão do exposto, o fator Neuroticismo foi decomposto pelo inventário de personalidade NEO-PI-R nas seguintes facetas: Ansiedade, Hostilidade, Depressão, Autoconsciência, Impulsividade e Vulnerabilidade.

De acordo com o estudo de Chmielewski et al. (2011), as facetas do NEO-PI-R foram particularmente efetivas em capturar as dimensões do TPB, predizendo quase $50 \%$ da variância em cada dimensão. Alguns estudos vêm sendo realizados no Brasil utilizando escalas que avaliam individualmente cada um dos cinco fatores por meio de suas facetas. Foram realizados estudos com as escalas fatoriais já validadas de SocializaçãoEFS, Extroversão-EFE e Neuroticismo-EFN (Nunes, 2007; Nunes, Hutz, \& Giacomoni, 2009; Trentini et al, 2009; Zanon \& Hutz, 2009). Contudo, não há, na literatura brasileira, estudos que avaliem as facetas do 
fator Neuroticismo por meio da Escala Fatorial de Neuroticismo (EFN) em pacientes com Transtorno da personalidade borderline.

A Escala Fatorial de Neuroticismo (EFN), desenvolvida por Nunes e Hutz (2001), é um instrumento que avalia especificamente a dimensão da personalidade denominada Neuroticismo/Estabilidade Emocional no modelo dos Cinco Grandes Fatores. Ela foi criada com base em afirmações que se relacionam com sintomas usualmente associados com Transtornos de Humor e com Transtornos da Personalidade. Na sua versão final, o teste é composto por 82 itens distribuídos em quatro subescalas: Escala de Vulnerabilidade, Escala de Ansiedade, Escala de Depressão e Escala de Desajustamento Psicossocial.

Diferentes argumentos corroboram o uso combinado de instrumentos que oportunizem avaliações categóricas e dimensionais. Levando em consideração o fato de que há um crescente corpo de pesquisa indicando que a combinação de avaliações categóricas e dimensionais otimizam a predição de importantes resultados psicossociais do paciente (Verheul, 2005; Krueger \& Eaton, 2010) e também a evidência de que clínicos podem se beneficiar de uma avaliação mais completa da personalidade, a qual foque na complexidade da psicopatologia e considere os traços problemáticos para intervenção e tratamento (Skodol et al., 2011; Fowlera et al., 2015), pretende-se, com este estudo, avaliar a diferença entre os sexos e a concordância e utilidade de uma avaliação dimensional da personalidade de pacientes com TPB por meio da EFN em confirmação com a avaliação categórica proposta pelo instrumento Borderline Symptoms List (BSL-23). Será investigado se as mulheres terão pontuação mais alta em Neuroticismo do que homens, visto que estudos anteriores demonstraram haver uma diferença significativa, inclusive em amostras de pacientes com o Transtorno Borderline (Maso \& Feitosa, 2013; Distel et al., 2009). Dessa forma, objetiva-se investigar a relevância das facetas do fator Neuroticismo em pacientes com marca da sintomatologia, como ocorre no Transtorno da Personalidade Borderline, sobretudo tratando-se de pacientes hospitalizados.

Pretende-se diagnosticar entre os pacientes aqueles que sofreram algum tipo de abuso (sexual, físico ou emocional) por meio de entrevista clínica e dentre estes, avaliar, por meio da Structured Clinical Interview for DSM-IV Axis I Disorders (SCID-I), aqueles que possam vir a apresentar Transtorno de Estresse Pós-Traumático (TEPT). Espera-se que a população clínica apresente sintomas moderadamente significativos quando aplicada a BSL-23; que pontue mais alto nas facetas do fator Neuroticismo quando comparada com a população da amostra de normatização do teste; e que apresente sintomas de TEPT na ocorrência de experiências traumáticas vividas na infância.

\section{Método}

\section{Participantes}

A amostra de pacientes com Transtorno da personalidade borderline (TPB) $(n=40)$ teve como idade mínima 18 anos e máxima 53 anos $(M=26,7 ; D P=7,75)$ e $72,5 \%$ da amostra era do sexo feminino (29 mulheres e 11 homens). Foram identificados 63 pacientes com Transtorno da Personalidade Borderline durante o período de estudo na clínica. Destes, cinco se recusaram a participar do estudo e 18 apresentaram critérios de exclusão.

Como critério de inclusão, admitidas as comorbidades, foi verificado por meio da BSL-23 (Borderline Symptoms List-23) se o Transtorno da Personalidade Borderline assumia maior dano na vida do paciente, sendo os demais diagnósticos considerados secundários em termos de prejuízo. Os critérios de exclusão foram definidos com o objetivo de assegurar a compreensão dos itens das escalas e evitar sintomatologia confundidora. Compreenderam: escolaridade inferior ao ensino médio incompleto, Transtorno de Humor Bipolar tipo I (avaliado pela Structured Clinical Interview for DSM-IV Axis I Disorders, SCID-I), diagnósticos de demência, esquizofrenia, dependentes químicos com menos de sete dias de abstinência e psicoses agudas.

\section{Procedimentos}

Inicialmente, foi feito contato com instituições para explicar a pesquisa. Solicitou-se autorização para a realização dos procedimentos na instituição. Após a autorização, foi solicitado aos terapeutas indicação de pacientes. A amostra foi recrutada por conveniência, a partir da suspeita de TPB pela indicação de profissionais da área de saúde mental da instituição que colaborou com o estudo, bem como por meio da busca ativa em prontuários da unidade de internação psiquiátrica.

Passada essa etapa, foi realizado contato inicial com esses pacientes, solicitando participação no estudo. Os participantes foram, desde o início, informados sobre o objetivo e a forma de coleta e análise dos dados. Não se buscou ocultar nenhum tipo de informação, uma vez que o conhecimento sobre os objetivos e detalhes do projeto desta pesquisa não introduziriam viés importante nos dados. Pelo contrário, contribuiriam para que os participantes se envolvessem adequadamente nas diversas fases do projeto. Apresentou-se, então, o Termo de Consentimento Livre e Esclarecido. No termo, estava destacado o direito de interromperem a participação na pesquisa no momento que desejassem, sem prejuízo algum, bem como a interrupção da participação, caso o resultado da avaliação pela SCID-II fosse negativo para TPB. Obtido o consentimento, dava-se início à aplicação dos instrumentos.

A privacidade dos participantes e a confidencialidade dos dados foi garantida, sendo que os casos receberam um número para facilitar a identificação de todo material 
coletado. Os questionários foram respondidos em local adequado para a testagem e o mesmo aplicador esteve presente na aplicação de todos os sujeitos. Foi aplicada inicialmente a Structured Clinical Interview for DSM-IV Axis II Disorders, SCID-II, para avaliar a confiabilidade diagnóstica para o Transtorno da Personalidade Borderline, a Structured Clinical Interview for DSM-IV Axis I Disorders, SCID-I, e a BSL-23. Depois da confirmação do diagnóstico, foi aplicada a Escala Fatorial de Neuroticismo (EFN). O uso desses inventários, instrumentos e entrevistas semiestruturadas baseia-se em estudos como o de Widiger e Samuel (2005) que demonstraram a eficácia do uso de múltiplos métodos para avaliações dos transtornos de personalidade. O tempo aproximado de aplicação foi de 150 minutos.

Esta pesquisa foi aprovada pelo Comitê de Ética e Pesquisa da UFRGS e está de acordo com a Resolução $n^{\circ}$. 466/2012 do Conselho Nacional de Saúde.

\section{Instrumentos}

\section{Questionário Sócio Demográfico \\ Structured Clinical Interview for DSM-IV Axis II Disorders, SCID-II (First et al., 1997). Trata-se} de uma entrevista semiestruturada, com base nos critérios diagnósticos do DSM-IV- TR (American Psychological Association - APA, 2003), que verifica a presença ou não dos sintomas explicitados no manual diagnóstico para Transtorno da Personalidade Borderline e outros transtornos de personalidade. Existem evidências na literatura acerca da consistência entre a SCID-II e a observação clínica (Maffei et al., 1997).

Structured Clinical Interview for DSM-IV Axis I Disorders, SCID-I (First et al., 1997). Trata-se de uma entrevista semiestruturada, com base nos critérios diagnósticos do DSM-IV-TR (APA, 2003), que verifica a presença ou não dos sintomas dos transtornos de EIXO-I presentes no manual.

Escala Fatorial de Neuroticismo, EFN (Hutz \& Nunes, 2001). A Escala Fatorial de Neuroticismo (EFN) é um teste psicométrico que mede o desajustamento emocional das pessoas no modelo dos Cinco Grandes Fatores (CGF) da personalidade. O instrumento contém 82 itens dispostos em escala Likert de 1 a 7 que avaliam quatro subfatores que integram o fator da personalidade Neuroticismo: vulnerabilidade, desajustamento psicossocial, ansiedade e depressão. $\mathrm{O} \alpha$ de Cronbach da Escala de Neuroticismo foi de $\alpha=0,94$, idêntico ao $\alpha$ de Cronbach original.

Borderline Symptoms List, BSL-23 (Bohus et al., 2009). Essa escala possibilita avaliar a sintomatologia do Transtorno da Personalidade Borderline em pessoas que possuem o transtorno. É um instrumento de autoavaliação com 23 itens onde a pessoa seleciona, em uma escala de 0 a 4 , a concordância com afirmativas sobre como ela sentiu os sintomas borderline na semana anterior à avaliação $(0=$ nada; $1=$ um pouco; $2=$ consideravelmente; 3 = muito; 4 = muito fortemente). $O$ instrumento possui alfa de Cronbach de 0,93 e está em processo de validação no Brasil.

\section{Procedimento para Análise dos Dados}

As variáveis quantitativas foram descritas por média e desvio padrão ou mediana e amplitude interquartílica, dependendo da distribuição dos dados. A normalidade dos dados foi avaliada pelo teste de Shapiro-Wilk e a homogeneidade de variâncias foi avaliada pelo teste de Levene. Em caso de distribuição simétrica e homogeneidade, testes paramétricos foram aplicados. Na quebra dessas suposições, testes não paramétricos foram utilizados. Dessa forma, foi realizada uma comparação das médias dos dados normativos das facetas e do total da EFN entre os sexos pelo Teste $t$ Student. Em caso de assimetria, o Teste $U$ de Wilcoxon-Mann-Whitney foi utilizado. Utilizou-se correlação de Pearson para avaliar a associação entre as variáveis contínuas de distribuição normal e de Spearman para as com distribuição assimétrica. $\mathrm{O}$ Teste Qui-quadrado de tendência linear foi aplicado para avaliar as associações entre as variáveis categóricas ordinais e o qui-quadrado complementado pela Razão de Prevalências (RP) para as nominais. Para comparar as facetas da EFN entre si, a Análise de Variância (ANOVA) de medidas repetidas foi aplicada. Em caso de significância estatística, o teste de Bonferroni foi utilizado na complementação da ANOVA. É importante ressaltar que todas as análises foram realizadas considerando os dados normativos, e não os dados brutos. O nível de significância adotado foi de $5 \%(p \leq 0,05)$ e as análises foram realizadas no programa SPSS versão 21.0.

\section{Resultados}

Observou-se que 5\% da amostra não apresentou nenhum transtorno de Eixo I identificável, 35\% apresentou um transtorno; $22,5 \%$ dois e 30\% apresentou três transtornos. Dentre as comorbidades, $40 \%$ da amostra apresentou Depressão Maior, Bipolaridade tipo II (17,5\%), Ciclotimia (12\%), Transtorno Alimentar (35\%), Transtorno de Ansiedade (20\%) e Transtorno de Estresse Pós-Traumático (18\%). Com relação aos transtornos de personalidade, $15 \%$ apresentou apenas o Transtorno de Personalidade Borderline. O restante da amostra, embora pudesse ter comorbidade com outros transtornos de personalidade, principalmente do cluster $\mathrm{B}$, teve o Transtorno Borderline como o mais prejudicial e significativo, conforme medido pela pontuação na BSL-23.

A média da população geral na Escala Fatorial de Neuroticismo (EFN) foi dimensionada para 100 pontos com um desvio padrão de 20. A média de pontuação desses pacientes na Escala Fatorial de Neuroticismo foi de $122,39 \pm 12,00$. O mínimo de pontuação obtida foi 89,30 e o máximo, 135,70. A média de pontuação de homens 
$(121,71 \pm 7,71)$ e mulheres $(122,65 \pm 13,38)$ foi bastante próxima. Conforme encontrado no Teste-t, a diferença entre a pontuação de homens e de mulheres na EFN não foi significativa $[t=(23)=-0,218, p=0,20]$, tamanho do efeito 0,08 .

Também não há diferença significativa entre os sexos nas pontuações de cada uma das subescalas que compõem a EFN (ver Tabela 1). Quando comparadas as facetas da EFN entre si dentro de cada sexo, observa-se diferença significativa tanto para homens $(p=0,014)$ quanto para mulheres $(p=0,002)$. Para homens houve, uma pontuação significativamente menor na subescala desajustamento social, quando comparado à ansiedade e depressão. Para as mulheres a pontuação foi significativamente mais elevada para depressão quando comparado às demais subescalas.

Tabela 1

Comparação entre os Sexos nas Facetas da EFN (Escores Padronizados)

\begin{tabular}{|c|c|c|c|c|c|c|}
\hline \multirow[b]{2}{*}{ Variáveis } & \multicolumn{2}{|c|}{ Homem } & \multicolumn{2}{|c|}{ Mulher } & \multirow[b]{2}{*}{$p<$} & \multirow[b]{2}{*}{ ES } \\
\hline & Média & $\begin{array}{l}\text { Desvio } \\
\text { Padrão }\end{array}$ & Média & $\begin{array}{l}\text { Desvio } \\
\text { Padrão }\end{array}$ & & \\
\hline 1. Vulnerabilidade & 30,8 & 3,5 & 29,8 & 4,2 & 0,479 & 0,25 \\
\hline 2. Desajustamento Psicossocial & 27,5 & 2,7 & 29,1 & 4,6 & 0,180 & 0,39 \\
\hline 3. Ansiedade & 31,0 & 2,7 & 30 & 4,1 & 0,968 & 0,03 \\
\hline 4. Depressão & 32,4 & 2,8 & 32,8 & 3,6 & 0,743 & 0,12 \\
\hline
\end{tabular}

Nota. ${ }^{*} p<0,05$

Tabela 2

Pontuação dos Escores Percentílicos das Facetas da EFN

\begin{tabular}{lcccc}
\hline & Variáveis & Menos de 30 & Entre 30 e 70 & Mais de 70 \\
\hline 1. Vulnerabilidade & $5 \%$ & $20 \%$ & $75 \%$ \\
2. Desajustamento & $5 \%$ & $25 \%$ & $70 \%$ \\
3. Ansiedade & $2,5 \%$ & $20 \%$ & $77,5 \%$ \\
4. Depressão & - & $5 \%$ & $95 \%$ \\
\hline
\end{tabular}

Em geral, as médias nas facetas da EFN mostraram-se bastante altas, acima de $70 \%$ em todas as subescalas, (ver Tabela 2). Segundo o manual, um valor maior que 70 pontos ou menor que 30 encontrado em algum desses escores percentílicos pode indicar uma configuração de distúrbio psicológico. O presente estudo, portanto, apresenta essa característica de alteração em no mínimo $75 \%$ dos sujeitos considerando todas as subescalas.

Na pontuação da BSL-23, a diferença entre homens e mulheres não foi significativa e os pacientes afirmaram apresentar consideravelmente os sintomas do Transtorno Borderline (homens 2,17 $\pm 0,49$; mulheres 2,20 \pm 0,67; $p=0,92)$. A correlação entre a EFN e a BSL-23 foi forte $(r=0,62)$. Ainda, encontrou-se uma correlação forte entre a BSL-23 e a faceta de ansiedade $(r=0,61 ; p \leq 0001)$ e a faceta vulnerabilidade $(r=0,64 ; p \leq 0001)$. As facetas depressão e desajustamento psicossocial demonstraram correlações moderadas com a BSL-23, respectivamente $r=0,34, p=0,035$ e $r=0,35, p=0,025$. Correlações de moderadas a fortes foram obtidas entre as facetas da escala de Neuroticismo e a escala total (EFN Total): a faceta vulnerabilidade demonstrou correlação de $r=0,82$, a de desajustamento psicossocial $r=67$, a de ansiedade $r=0,86$ e a de depressão $r=0,71$. A faceta vulnerabilidade e o escore total da EFN se correlacionaram de forma positiva e significativa com o número de comorbidades $\left(r_{s}=0,44, p=0,005\right.$ e $r_{s}=0,35, p=0,026$, respectivamente). Todas as correlações significativas são de moderadas a forte, (ver Tabela 3). Não houve associações significativas entre as subescalas da EFN e os transtornos de Eixo I, exceto na associação entre abuso e TEPT, que se mostrou significativa, $(\mathrm{RP}=5,25$; IC95\%=1,00-31,9; $p=0,011)$, sendo que pacientes que apresentaram abuso têm uma prevalência 5,25 maior de desenvolverem TEPT quando comparados aos que não tiveram situações de abuso. Também foi encontrada associação linear significativa entre a faceta vulnerabilidade e o Transtorno de Personalidade Evitativa $\left(\chi_{\text {linear }}^{2}=4,11\right.$; $g l=1 ; p=0,043)$ e a faceta desajustamento psicossocial e o Transtorno de Personalidade Histriônica $\left(\chi_{\text {linear }}^{2}=7,86\right.$; $g l=1 ; p=0,005)$, sendo que o percentual do transtorno aumenta à medida que aumenta a classificação do paciente em percentil. 
Tabela 3

Correlações entre a BSL-23, EFN-Total, Facetas da EFN e Comorbidades

\begin{tabular}{|c|c|c|c|c|c|c|}
\hline Variáveis & (1) & (2) & (3) & (4) & (5) & (6) \\
\hline 1. BSL-23 & - & - & - & - & - & - \\
\hline 2. EFN & $0,62^{* * *}$ & - & - & - & - & - \\
\hline 3. EFN Vulnerabilidade & $0,64^{* * *}$ & $0,82^{* *}$ & - & - & - & - \\
\hline 4. EFN Desajustamento & $0,35^{*}$ & $0,67^{* *}$ & $0,41^{* *}$ & - & - & - \\
\hline 5. EFN Ansiedade & $0,61^{* * *}$ & $0,86^{* *}$ & $0,67^{* * *}$ & $0,52^{* *}$ & - & - \\
\hline 6. EFN Depressão & $0,34^{*}$ & $0,71^{* *}$ & $0,46^{* *}$ & 0,26 & $0,62^{* * *}$ & - \\
\hline 7. Comorbidades & 0,29 & $0,35^{*}$ & $0,44^{* *}$ & 0,08 & 0,25 & 0,17 \\
\hline
\end{tabular}

Nota. ${ }^{*} p<0,05 ;{ }^{* *} p<0,01 ;{ }^{* * *} p<0,001$

\section{Discussão}

O objetivo deste trabalho foi investigar as diferenças entre os sexos na Escala Fatorial de Neuroticismo (EFN) e em suas facetas em 40 pacientes com Transtorno da Personalidade Borderline internados em uma clínica psiquiátrica bem como a ocorrência de TEPT nesses pacientes. Segundo o manual da EFN (Hutz \& Nunes, 2001), não é possível elaborar pontos de corte para a discriminação de casos dentro das concepções de "normalidade versus patologia”, mas acredita-se que escores muito altos ou muito baixos devam indicar alguns tipos de transtornos da personalidade. A média dos pacientes avaliados foi de 122,39, o que corrobora com os dados indicados pelo manual, já que a média da população geral na Escala Fatorial de Neuroticismo (EFN) é dimensionada para 100 pontos com um desvio padrão de 20.

Em diversos estudos sobre as diferenças entre os sexos nas facetas dos fatores da EFN (Nunes, 2000; Serafini, 2008; Trentini et al., 2009; Zanon, 2012), homens pontuaram mais em desajustamento psicossocial e depressão enquanto mulheres pontuaram mais em depressão e ansiedade, o que corrobora os achados do presente estudo. Não houve diferença significativa entre homens e mulheres na pontuação geral de Neuroticismo, o que vai de encontro a pesquisas anteriormente realizadas (Maso \& Feitosa, 2013; Distel et al., 2009). Todas as facetas de Neuroticismo se correlacionaram com a BSL-23 o que traz evidência favorável à pertinência do uso da EFN para o diagnóstico de TPB, bem como para a compreensão dos sintomas desse complexo transtorno.

No estudo realizado por Samuel e Widiger (2008), os pacientes com Transtorno de Personalidade Borderline tiveram a maior correlação na faceta depressão $(r=0,50$, $p<0,05)$ do que entre todas as outras facetas, o que vai ao encontro dos dados encontrados. Outro fator significativo é que a maioria dos pacientes apresentou comorbidade com Depressão Maior (40\%), o que pode igualmente explicar esses achados. Contudo, em artigo publicado no JAMA Psychiatry pelos pesquisadores do Genetics of Personality Consortium - GPC (2015) foi identificada uma relação genética entre Neuroticismo e Depressão. O Gene MAGI1 seria um dos responsáveis por várias doenças psiquiátricas, dentre elas Depressão Maior, e este explicaria $15 \%$ da variância em Neuroticismo.

As comorbidades se correlacionaram de forma significativa com a faceta vulnerabilidade e esta se correlacionou de forma significativa com todas as outras facetas. Escores altos em Desajustamento Psicossocial sugerem Transtornos de Personalidade Antissocial e Borderline. Apesar de apresentarem resultados lineares, essas associações não foram significativas. Entretanto, escore alto em Desajustamento Psicossocial mostrou-se correlacionado com personalidade histriônica $(p=0,005)$, transtorno que constitui o mesmo grupo dos Transtornos Borderline e Antissocial, o cluster B. A associação entre desajustamento social e personalidade histriônica pode ser devido à inadequação nos relacionamentos interpessoais que as pessoas com personalidade histriônica possuem.

No presente estudo, obteve-se uma associação significativa entre situações de abuso e a ocorrência de TEPT e os dados demonstraram que é cinco vezes maior a ocorrência de TEPT entre os sujeitos que foram abusados. 90,9\% daqueles que sofreram abuso pontuaram mais de 70 na faceta de Ansiedade da EFN. Mais da metade da amostra (55\%) teve alguma situação de abuso físico, sexual e/ou emocional no passado, o que se aproxima de achados anteriores em uma amostra de pacientes como TPB do Rio Grande do Sul, onde a taxa de abuso foi de $60,8 \%$ (Schestatsky, 2005). Dos que sofreram abuso, 31,8\% apresentaram Transtorno de Estresse Pós-Traumático. A pesquisa de Collin-Vézina \& Hébert (2005), apontou a prevalência de $30 \%$ e $40 \%$, respectivamente, de sintomas de dissociação e TEPT em meninas vítimas de abuso sexual. Alta prevalência de ansiedade, depressão, TEPT e Transtorno de Personalidade Borderline foram encontrados em mulheres que sofreram abuso sexual na infância (MacMillan et al., 2001; Grassi-Oliveira, 2005; Linehan,2009). No estudo de Cutajar et al. (2010), meninas abusadas demonstraram cerca de 7,6 vezes mais chance de desenvolver TPB do que as que não sofreram abuso, o que pode explicar a 
alta incidência de abuso na amostra escolhida e ser mais um indício de que esse pode ser um fator importante na etiologia do transtorno.

É necessário investigar a pertinência do uso dessas escalas, sobretudo da EFN, na população clínica, considerando que a sua normatização para uso na população brasileira ocorreu com participantes eutímicos. Além disso, sugere-se um estudo com populações clínicas maiores e outros que aprofundem as relações entre TEPT, abuso e a ocorrência de depressão nesses pacientes. Dado que este estudo realizou análises bivariadas e contou com uma amostra de tamanho reduzido, pode-se empregar para pesquisas futuras o emprego de análises fatoriais, com amostras maiores, como uma estratégia para analisar de forma mais precisa a variância partilhada entre esses instrumentos.

\section{Referências}

American Psychological Association (2003). Manual Diagnóstico e Estatístico de Transtornos Mentais DSM-IV-TR (4a Ed.). Porto Alegre: Artmed. American Psychiatric Association (2013). Desk reference to the diagnostic criteria from DSM-5. Washington: DC.

Bagby, R. M., Costa, P. T. J., \& Widiger, T. (2005). DSM-IV personality disorders and the five-factor model of personality: A multi-method examination of domain and facet-level predictions. European Journal of Personality, 19, 307-324. doi: 10.1002/per.563" 10.1002/per.563

Bohus, M., Kleindienst, N., Limberger, M. F., Stieglitz, R. D., Domsalla, M., Chapman, A. L., Steil, R., Philipsen, A., \& Wolf, M. (2009). The short version of the borderline symptom list (BSL-23): Development and initial data on psychometric properties. Psychopathology, 13, 32-39. doi: 10.1159/000173701

Brown, T. A., \& Barlow, D. H. (2009). A proposal for a dimensional classification system based on the shared features of the DSM-IV anxiety and mood disorders: Implications for assessment and treatment. Psychological Assessment, 21, 256-271. doi: 10.1037/a0016608

Clarck, L. A. (2007). Assesment and diagnosis of personality disorder: Perennial issues and an emerging reconceptualization. Annual Review of Psychology, 19, 227-257. doi: 10.1146/annurev.psych.57.102904.190200

Chiu, A. (2013). Validity of Proposed DSM-5 Personality Disorder Trait Domains (Tese de Doutorado). St. John's University, New York, USA.

Chmielewski, M., Bagby, R. M., Quilty, L. C., Paxton, R., \& McGee, S. (2011). A (re)-evaluation of the symptom structure of borderline personality disorder. La Revue Canadian en Psychiatrie, 56(9), 530-553. doi: 10.1177/0706743711105600904"10.1177/070674371105600904

Collin-Vézina, D., \& Hébert, M. (2005). Comparing dissociation and PTSD in sexually abused school-aged girls. Journal of Nervous and Mental Disease, 193(1), 47-52. doi: 10.1097/01.nmd.0000149218.76592.26

Cutajar, M. C., Mullen, P.E., Ogloff, J. R., Thomas, S. D., Wells, \& D. L., Spataro, J.(2010) Psychopathology in a large cohort of sexually abused children followed up to 43 years. Child Abuse Neglect, 34, 813-822. doi: 10.1016/j.chiabu.2010.04.004"10.1016/j.chiabu.2010.04.004

Fowlera, J. C., Sharpa, C., Kalpakcia, A., Madana, A., Clappd, J., Allena, J. G., Frueha, C., \& Oldhama, J. M. (2015). A dimensional approach to assessing personality functioning: Examining personality trait domains utilizing DSM-IV personality disorder criteria. Comprehensive Psychiatry, 56, 75-84. doi: 10.1016/j.comppsych.2014.09.001"10.1016/j.comppsych.2014.09.001

Distel, M. A., Trull, T. J., Willemsen, G., Vink, J. M., Derom, C. A., Lynskey, M., \& Boomsma, D. I. (2009). The five-factor model of personality and borderline personality disorder: A genetic analysis of comorbidity. Biological Psychiatry, 66(12), 1131-1138. doi: 10.1016/j.biopsych.2009.07.017

Gabbard, G. O. (2000). A neurobiologically informed perspective on psychotherapy. The British Journal of Psychiatry, 177(2), 117-122. doi: 10.1192/bjp.177.2.117"10.1192/bjp.177.2.117

Genetics of Personality Consortium (2015). Meta-analysis of genome-wide association studies for neuroticism, and the polygenic association with major depressive disorder. Jama Psychiatry, 72(7), 642-650. doi: 10.1001/jamapsychiatry.2015.0554

Grant, B. F., Chou, S. P., Goldstein, R. B., Huang, B., Stinson, F. S., Saha, T. D., ... \& Ruan, W. J. (2008) Prevalence, correlates, disability, and comorbidity of DSM-IV borderline personality disorder: Results from the Wave 2 National Epidemiologic Survey on Alcohol and Related Conditions. The Journal of Clinical Psychiatry, 69(4), 533-545. Recuperado de https://www.ncbi.nlm.nih.gov/pmc/articles/ PMC2676679/

Grassi-Oliveira, R. (2005). Maus-tratos na infância: Instrumentos de avaliação e estudo de associação com transtorno de estresse pós-traumático e sintomas psiquiátricos gerais em adultos (Dissertação de mestrado não publicada). Programa de Pós-Graduação em Psicologia, Pontifícia Universidade Católica do Rio Grande do Sul, Porto Alegre.

Gore, W. L., \& Widiger, T. A. (2013). The DSM-5 dimensional trait model and five-factor models of general personality. Journal of Abnormal Psychology, 122(3), 816-821. doi: 10.1037/a0032822"10.1037/a0032822

Gunderson, J. G. (2009). Borderline personality disorder: Ontogeny of a diagnosis. American Journal Psychiatry, 166(5), $530-539$.

Hutz, C. S., \& Nunes, C. H. S. S. (2001). Escala fatorial de ajustamento emocional /neuroticismo- EFN. São Paulo: Casa do Psicólogo.

Krueger R. F., \& Eaton N. R. (2010). Personality traits and the classification of mental disorders: Toward more complete integration in DSM-5 and an empirical model of psychopathology. Personality Disorders, 1(2), 97-118. doi: 10.1037/a0018990

Krueger, R. F., Eaton, N. R., Clark, L. A., Watson, D., Markon, K. E., Derringer, J., Skodol, A., \& Livesley, J. (2011). Deriving an Empirical Structure of Personality Pathology for DSM-5. Journal of Personality Disorders, 25(2), 170-191. doi: 10.1521/pedi.2011.25.2.170

Leichsenring, F., Leibing, E., Kruse, J., New, A. S., \& Leweke, F., (2011). Borderline personality disorder. Lancet, 377(9759), 74-84.

Linehan, M. M. (2009). Terapia cognitivo-comportamental para transtorno da personalidade Borderline: Guia do terapeuta. Porto Alegre: Artmed.

Livesley, W. J. (2007). A framework for integrating dimensional and categorical classifications of personality disorder. Journal of Personality Disorders, 21(2), 199-224. doi: 10.1521/pedi.2007.21.2.199"10.1521/pedi.2007.21.2.199

MacMillan, H. L., Fleming, J. E., Streiner, D. L., Lin, E., Boyle, M. H., Jamieson, E., Duku, E. K., Walsh, C. A., Wong, M. Y., \& Beardslee, W. R. (2001). Childhood abuse and lifetime psychopathology in a community sample. American Journal of Psychiatry, 158(11), 18781883. doi: 10.1176/appi.ajp.158.11.1878 
Maffei, C., Fossati, A., Agostoni, I., Barraco, A., Magnato, M., Deborah, D., Namia, C, Novella, L., \& Petrachi, M. (1997). Interrater reliability and internal consistency from DSM-IV Axis II personality disorders (SCID-II), version 2.0. Journal of Personality Disorders, 11(3), 279-284. doi: 10.1521/pedi.1997.11.3.279"10.1521/pedi.1997.11.3.279

Markon, K. E., Krueger, R. F., \& Watson, D. (2005) Delineating the structure of normal and abnormal personality: An integrative hierarchical approach. Journal of Personality and Social Psychology, 88(1), 139-157. doi: 10.1521/pedi.1997.11.3.279"10.1521/pedi.1997.11.3.279

Maso, M. D., \& Feitosa, F. B. (2013). Um estudo comparativo entre dados sociodemográficos e neuroticismo. Estudos e Pesquisas em Psicologia, 13(3), 1182-1197. Recuperado de http://pepsic.bvsalud.org/scielo.php?script=sci_abstract\&pid=S1808-42812013000300019

McCrae, R. R., \& John, O. P. (1992). An introduction to the Five-Factor Model and its applications. Journal of Personality, 60(2), $175-216$. doi: 10.1037/0021-843X.109.4.733

Morey, L. C., \& Zanarini, M. C. (2000). Borderline personality: Traits and disorder. Journal of Abnormal Psychology, 109(4), 733-773. doi: 10.1037/0021-843X.109.4.733

Mosquera, D., Gonzalez, A., \& Leeds, A. M., (2014). Early experience, structural dissociation, and emotional dysregulation in borderline personality disorder: The role of insecure and disorganized attachment. Borderline Personality Disorder and Emotion Dysregulation, 1(15), 6673-6615. doi: 10.1186/2051-6673-1-15

Nunes, C. H. S. S. (2007). Lançamento da Escala Fatorial de Extroversão (EFEx) e Escala Fatorial de Socialização (EFS). Avaliação Psicológica, 6(1), 103-106. Recuperado de http://pepsic.bvsalud.org/scielo.php?script=sci_arttext\&pid=S1677-04712007000100013

Nunes, C. H. S., Hutz, C. S., \& Giacomoni, C. H. (2009). Associação entre bem estar subjetivo e personalidade no modelo dos cinco grandes fatores. Avaliação Psicológica, 8(1), 99-108. Recuperado de http://pepsic.bvsalud.org/scielo.php?script=sci_arttext\&pid=S1677-04712009000100009

O'Connor, B. P. (2002). The search for dimensional structure differences between normality and abnormality: A statistical review of published data on personality and psychopathology. Journal of Personality and Social Psychology, 83(4), 962-982. doi: 10.1037/0022-3514.83.4.962

Pervin, L. A., \& John, O. P. (2004). Personalidade: Teoria e Pesquisa. Porto Alegre: Artmed.

Samuel, D. B., \& Widiger, T. A. (2008). A meta-analytic review of the relationships between the five-factor model and DSM-IV-TR personality disorders: A facet level analysis. Clinical Psychology Review, 28(8), 1326-1342. doi: 10.1016\%2Fj.cpr.2008.07.002

Saulsman, L. M., \& Page, A. C. (2004). The five-factor model and personality disorder empirical literature: a meta-analytic review. Clinical Psychological Review, 23(8), 1055-1085. doi: 10.1016/j.cpr.2002.09.001"10.1016/j.cpr.2002.09.001

Serafini, A. J. (2008). Satisfação de vida, rede de relações, coping, neuroticismo em adolescentes portadores e não portadores do vírus da imunodeficiência humana - HIV. (Tese de doutorado não publicada). Universidade Federal do Rio Grande do Sul, Porto Alegre.

Soloff, P., Fabio, A., Kelly, T., Malone, K., \& Mann J. (2005). High-lethality status in patients with borderline personality disorder. Journal of Personality Disorders, 19, 386-399. doi: 10.1521/pedi.2005.19.4.386

Schestatsky, S. D. (2005). Fatores ambientais e vulnerabilidade ao transtorno de personalidade borderline: Um estudo caso-controle de traumas psicológicos precoces e vínculos parentais percebidos em uma amostra brasileira de pacientes mulheres (Tese de doutorado). Universidade Federal do Rio Grande do Sul. Porto Alegre.

Skodol, A. E., Clark, L. A., Bender, D. S., Krueger, R. F., Morey, L. C., ... Verheul, R. (2011). Proposed changes in personality and personality disorder assessment and diagnosis for DSM-5 part I: description and rationale. Personality Disorder, 2(1),4-22. doi: 10.1037/a0021891

Torgersen, S. (2009). The nature (and nurture) of personality disorders. Scandinavian Journal of Psychology, 50(6),624-632. doi: 10.1111/j.14679450.2009.00788.x

Trentini, C. M., Hutz, C. S., Bandeira, D. R., Teixeira, M. A. P., Gonçalves, M. T. A., \& Thomazoni, A. R. (2009). Correlações entre a EFN - Escala Fatorial de Neuroticismo e o IFP - Inventário Fatorial de Personalidade. Avaliação Psicológica, 8(2), 209-217. Recuperado de http://pepsic.bvsalud.org/scielo.php?script=sci_arttext\&pid=S1677-04712009000200007

Verheul, R. (2005). Clinical utility for dimensional models of personality pathology. Journal of Personality Disorders, 19(3), 283-302. doi: 10.1521/pedi.2005.19.3.283

Yen, S., Shea, M. T., Battle, C. L., Johnson, D. M., Zlotnick, C., Dolan-Sewell, Skodol, A. E., Grilo, C. M., Gunderson, J. G., Sanislow, C. A., Zanarini, M. C., Bender, D. S., Rettew, J. B., \& McGlashan, T. H., (2002). Traumatic exposure and posttraumatic stress disorder in borderline, schizotypal, avoidant, and obsessive-compulsive personalitydisorders: Findings from the collaborative longitudinal personality disorders study. The journal of nervous and mental disease, 190(8), 510-518. doi: 10.1097/01.NMD.0000026620.66764.78

Zanon, C., \& Hutz, C. S. (2009). Propriedades psicométricas da Escala Fatorial de Neuroticismo e do Questionário de Ruminação e Reflexão. Avaliação Psicológica, 8(2), 279-281. Recuperado de http://pepsic.bvsalud.org/scielo.php?script=sci_arttext\&pid=S1677-04712009000200013

Zanon, C., Borsa, J. C., Bandeira, D. R., \& Hutz, C. S. (2012). Relações entre pensamēnto ruminativo e facetas do neuroticismo. Estudos de Psicologia (Campinas), 29(2), 173-181. Recuperado de http://www.academia.edu/17521273/Relações_entre_pensamento_ruminativo_e_ facetas do neuroticismo

Zimmerman, M., Rothschild, L., \& Chelminsky, I. (2005). The prevalence of DSM-IV personality disorders in psychiatric outpatients. American Journal of Psychiatry, 162(10), 1911-1918. doi: 10.1176/appi.ajp.162.10.1911

Widiger, T. A., \& Samuel, D. B. (2005). Evidence based assessment of personality disorders. Psychological Assessment, 17(3), 278-287. doi: 10.1037/1040-3590.17.3.278

Wischniewski, J., \& Brüne, M. (2013).How do people with borderline personality disorder respond to norm violations? Impact of personality factors on economic decision-making. Journal of Personality Disorders, 27(4), 531-546. doi: 10.1521/pedi_2012_26_036

recebido em setembro de 2016 aprovado em maio de 2017

\section{Sobre os autores}

Letícia Garibaldi Gasparetto é graduada em Psicologia pela UFRGS, tem especialização em Terapia Cognitivo-Comportamental pelo Intcc e Mestrado em Desenvolvimento Humano pela UFRGS. Atualmente é servidora do Tribunal Regional Federal da $4^{\text {a }}$ Região.

Claudio Simon Hutz é Psicólogo, Mestre e Doutor pela University of Iowa (USA) e Pós-Doutor na Arizona State University (USA). Atualmente é Professor Titular da Universidade Federal do Rio Grande do Sul (UFRGS) e Coordenador do Laboratório de Mensuração do PPG Psicologia da UFRGS. 